\title{
Alignment of Biological Apatite Crystallites in Peri-Implant Bone of Beagles
}

\author{
Kento Odaka $^{1,2, *}$, Satoru Matsunaga ${ }^{1,2}$, Masaaki Kasahara ${ }^{1,2, *}$, Takayoshi Nakano ${ }^{3}$, Masao Yoshinari ${ }^{1}$ \\ and Shinichi $\mathrm{Abe}^{2}$
}

\author{
${ }^{1}$ Division of Oral Implants Research, Oral Health Science Center, Tokyo Dental College, Tokyo 101-0061, Japan \\ ${ }^{2}$ Department of Anatomy, Tokyo Dental College, Tokyo 101-0061, Japan \\ ${ }^{3}$ Division of Materials and Manufacturing Science, Graduate School of Engineering, Osaka University, Suita 565-0871, Japan
}

\begin{abstract}
The jawbone exists in a special mechanical environment where it is subjected to functional pressure including occlusal force. The internal structure and bone strength of the jawbone might change as a result of tooth loss and implant placement. Therefore, the local bone quality must be evaluated to elucidate the load environment applied to peri-implant jawbone. The present study was conducted to clarify the nanostructural anisotropy of peri-implant jawbone of beagles by quantitatively evaluating biological apatite crystallite alignment. A total of 12 beagles were divided into four groups (dentate jawbone, edentulous jawbone 3 months after tooth extraction, edentulous jawbone 3 months after placing superstructure, and edentulous jawbone 12 months after placing superstructure). Each group comprised three samples. The fourth premolar was considered as the region of interest, with five measurement points in cortical bone around the implant. Each site was subjected to microbeam X-ray diffraction analysis to evaluate biological apatite crystallite alignment. The results revealed that biological apatite crystallite alignment had been lost in the samples taken after tooth extraction. Furthermore, in the dentate and post-implantation samples, preferential alignment was seen not only in the buccal alveolar region, but also in the infraorbital canal and nasal cavity floors. The results of this study suggested that changes of load environment resulting from dental implant treatment contribute to the expression of unique structural characteristics in peri-implant jawbone, which might affect regions relatively far from implants. [doi:10.2320/matertrans.M2016228]
\end{abstract}

(Received June 24, 2016; Accepted October 12, 2016; Published November 11, 2016)

Keywords: $\quad$ biological apatite crystallite, bone quality, peri-implant bone, microbeam X-ray diffraction, beagle

\section{Introduction}

The jawbone is a special bone in which functional pressure such as occlusal pressure is transmitted directly to the bone. Tooth dysfunction and removal are therefore known to greatly change not only the external form of alveolar bone, but also the internal structure and bone strength. Wada et al. reported morphological and elastic changes to the jawbone as a result of tooth milling ${ }^{1)}$. Meanwhile, Bassi et al. discussed the effects of chewing load on the jawbone after finding that edentulous sites have lower bone mineral density ${ }^{2}$. These changes of the jawbone may be attributed to decreases in the functional pressure applied via the teeth. Kingsmill et al. also reported significant differences depending on the presence or absence of teeth in the jawbone, but reported almost no decrease in bone mass with aging based on research on age-related and tooth loss-related changes to the jawbone ${ }^{3)}$. The functional pressure applied to the teeth is therefore considered vital in order to maintain jawbone homeostasis.

On the other hand, dental implants, which have become extremely popular in recent years, bond directly to the jawbone and therefore have a load transfer mechanism that greatly differs from that of teeth which bear load via periodontal ligament. Dental implants directly transmit load to the inside of the jawbone by osseointegration with the jawbone ${ }^{4)}$. A number of recent studies have revealed that the appropriate load encourages differentiation of peri-implant tissue and formation of new bone and contributes to maintaining homeostasis of the surrounding jawbone ${ }^{5-11)}$. On the other hand, some reports have described the possibility of lateral pressure or excessive load applied to the implant causing loss of marginal bone ${ }^{12-14)}$. While it is essential to predict the mechanical environment in peri-implant bone, evaluation of the invis-

*Graduate Student, Tokyo Dental College ible load transfer mechanism is considered difficult.

Bone is a passive locomotor apparatus and its structure is always optimized so that maximum strength can be exerted by the minimum amount of material ${ }^{15)}$. Bone strength evaluation is widely used as an indicator of the state of bone in bone diseases such as osteoporosis ${ }^{16}$. To date, bone strength has mainly been evaluated using bone mass, i.e., bone mineral density (BMD); however, bone quality factors have been referred to in recent years as a means of evaluating mechanical properties of bone that cannot be explained with bone mass ${ }^{17)}$. Of these bone quality factors, biological apatite (BAp) crystallite alignment exhibits strong resistance to compressive load and shows great promise in predicting load environment. BAp crystallite has a highly anisotropic structure of hexagonal unit cells and has been found to exhibit a high Young's modulus in the direction of preferential alignment ${ }^{18)}$.

Microbeam X-ray diffraction is a method used to obtain crystallization data of a material by measuring X-ray beams diffracted by the crystal lattice. It is a bone quality evaluation method that can be used to quantitatively evaluate BAp crystallite alignment in localized regions. Nakano et al. used this method to examine the ulnae, calvaria and mandibles of experimental animals and suggested site specificity of BAp crystallite alignment ${ }^{19,20)}$. Morioka et al. and Furuya et al. also used this method for detailed analysis of BAp crystallite alignment in human jawbone and reported that dentate mandibles exhibit a double structure on a nanoscale ${ }^{21,22)}$. Furthermore, Iwata et al. examined BAp crystallite alignment in edentulous jawbone and found that the preferential alignment of alveolar bone formed around the teeth was lost ${ }^{23)}$. However, few papers have discussed the nanoscale properties of jawbone around loaded implants. Much, therefore, remains unclear about the effects of artificially created load environments on structures surrounding the jawbone. Ohashi et al. predicted load transfer paths from the results of a three-di- 
mensional finite element analyses method and reported that compressive stress applied to the implant body propagates to distant sites via trabecular bone; however, this matter requires further investigation ${ }^{24)}$.

The present study was conducted to clarify the anisotropy of peri-implant bone and adjacent bone which is subjected to load, by quantitatively evaluating the crystallographic characteristics of BAp crystallite in peri-implant jawbone.

\section{Materials and Methods}

\subsection{Animals}

Twelve 12-month-old immunized male beagles weighing $10-15 \mathrm{~kg}$ and without physical abnormalities were divided into four groups after being reared for at least one week following receipt. These four groups were as follows: a dentate group euthanized without treatment (Dentate), an edentulous group euthanized 3 months after tooth extraction (Partially edentulous), an edentulous group in which implants were placed 3 months after tooth extraction, fitted with a superstructure after a 3-month healing period, and euthanized 3 months later $(3 \mathrm{M})$, and an edentulous group in which implants were placed 3 months after tooth extraction, fitted with a superstructure after a 3-month healing period, and euthanized 12 months later (12M; Fig. 1). The animal experiment was conducted in accordance with Tokyo Dental College Ethical Guidelines for Animal Research (A-06-0010-2).

\subsection{Surgical procedure}

First, the bilateral maxillary fourth premolars, first molars and second molars were extracted in order to place the implants and avoid the effect of occlusion in the 'not implanted' side. Prior to the experiment, the animals were premedicated with $0.2 \mathrm{mg} / \mathrm{kg}$ of ketamine hydrochloride $5 \%$ (Ketalar ${ }^{\circledR} 50$ for Intramuscular Injection; Sankyo Co., Ltd., Tokyo, Japan) administered intramuscularly, after which they were given an intravenous injection of $0.5 \mathrm{~mL} / \mathrm{kg}$ of pentobarbital sodium 5\% (Nembutal for Injection; Sumitomo Dainippon Pharma. Co., Ltd., Osaka, Japan) to induce general anesthesia. Infiltration anesthesia of the operative site was then performed using xylocaine 2\% (Xylocaine for Dental Use; Fujisawa Pharmaceutical Co., Ltd., Osaka, Japan). Thereafter, the area around the mouth and oral cavity were disinfected with benzalkonium chloride $0.5 \%$ (Benzalkonium Chloride Solution; Kozakai Pharmaceutical Co., Ltd., Tokyo, Japan) and surgery was performed. An incision was made in the gingival sulcus and the mucoperiosteal flap was reflected. The tooth root was split using a diamond disc and diamond bur under sterile saline irrigation, after which the bilateral maxillary fourth premolars, first molars and second molars were extracted using dental forceps. After a 3-month wound-healing period, a total of three implants were placed in the left maxillary molar region. Regular Neck (RN) SLA Implants (Straumann, Basel, Switzerland) with a diameter of $4.1 \mathrm{~mm}$ and length of $6 \mathrm{~mm}$ were used. An incision was then made into the alveolar crest as far as the periosteum and a mucoperiosteal flap was reflected to allow an implant cavity to be prepared using $\phi 2.2, \phi 2.8$ and $\phi 3.5$ pilot drills under sterile saline irrigation. Next, threads were formed under sterile saline irrigation and the fixture was placed. A healing abutment was fitted on the embedded implant and the operative field was washed with sterile saline, after which the mucoperiosteal flap was sutured to ensure that only the healing abutment was exposed. The implants were mesiodistally placed at regular intervals. Three months later, splinted crowns were fitted to each of the three implants. The superstructure was cast and manufactured using $12 \%$ gold-silver-palladium alloy (Kinpara S12; Ishifuku Metal Industry Co., Ltd., Tokyo, Japan). The general shape was not that of a natural dog tooth, but a simple cylindrical shape designed to remain as hygienic as possible (Fig. 2). The right molar region was checked for any malocclusion and occlusal contacts were created on the superstructure so that it came into contact with two or three points on the opposing tooth. The loading condition of surrounding bone in each group is shown in Fig. 3. After fitting the contacts, the animals were given solid food. The animals were euthanized 3 or 12 months later, and samples were resected from the maxillae.

\subsection{Measurement of biological apatite crystal orienta- tion}

The region of interest was established as the fourth premolar region, which had sufficient bone mass for X-ray diffraction. Five measurement points were set: point I on the infraorbital canal floor; points II and III on the nasal cavity floor

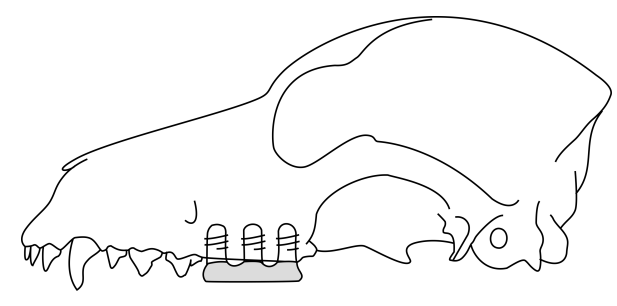

Fig. 2 Schematic drawing of the implant superstructure.

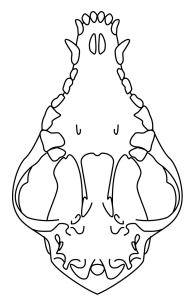

[ Dentate ]

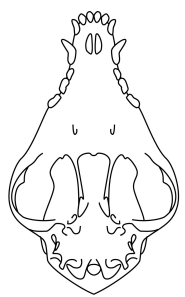

[ Partially edentulous ]

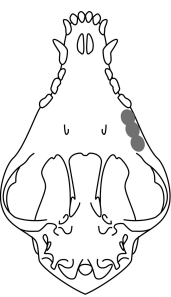

[ $3 \mathrm{M}$ ]

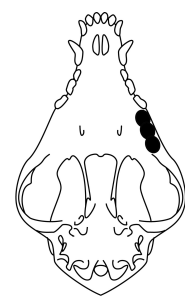

[ $12 \mathrm{M}$ ]

Fig. 1 Four experimental groups. Dentate: At the start of the experiment. Partially edentulous: 3 months after extraction. $3 \mathrm{M}$ : 3 months after fitting superstructure. $12 \mathrm{M}$ : 12 months after fitting superstructure. 

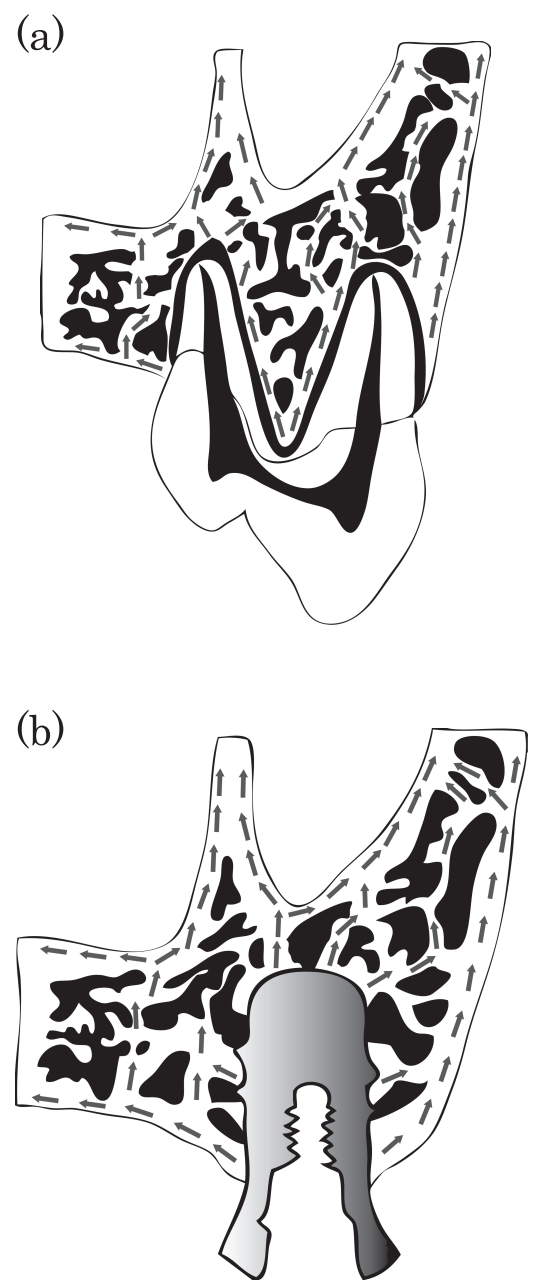

Fig. 3 Conceptual drawing of the load transfer path: (a) Dentate: (b) $3 \mathrm{M}$ and $12 \mathrm{M}$.

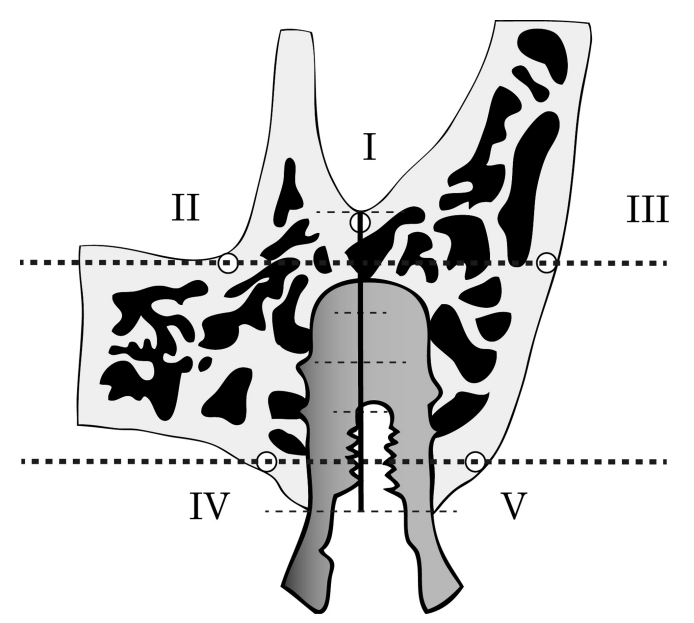

Fig. 4 Measurement sites in this experiment are shown. The dotted line represents the plane parallel to the occlusal plane. I: Cortical bone at the lowest point in the infraorbital canal floor. II: Cortical bone in the upper $1 / 6$ of the nasal cavity floor. III: Cortical bone in the upper $1 / 6$ on the buccal side. IV: Cortical bone in the lower $1 / 6$ on the palatal side. V: Cortical bone in the lower $1 / 6$ on the buccal side.

and buccal cortical bone, respectively, located on the occlusal plane in the upper $1 / 6$ of the distance from point I to the alveolar apex; points IV and $\mathrm{V}$ on the palatal and buccal cortical

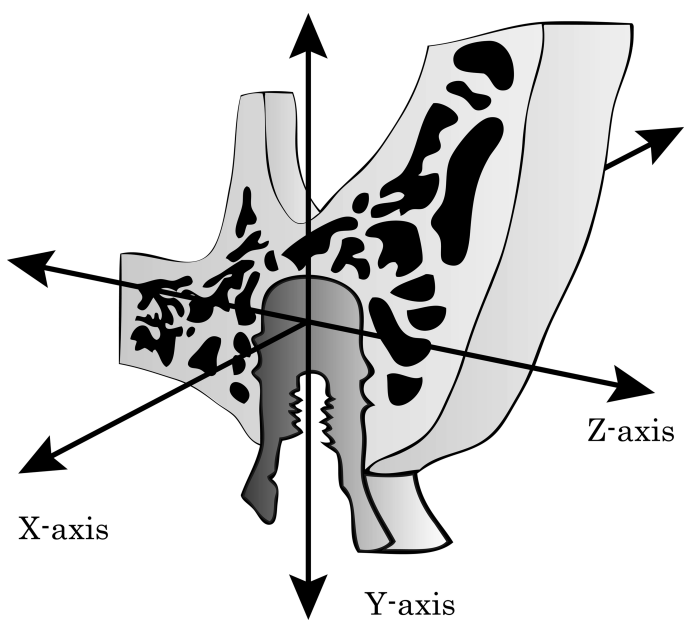

Fig. 5 Setting of the coordinate axes. Measurement directions were designated for the samples: the mesiodistal direction was placed along the $\mathrm{X}$-axis, the vertical occlusal plane along the $\mathrm{Y}$-axis, and the buccolingual direction along the Z-axis.

(a)

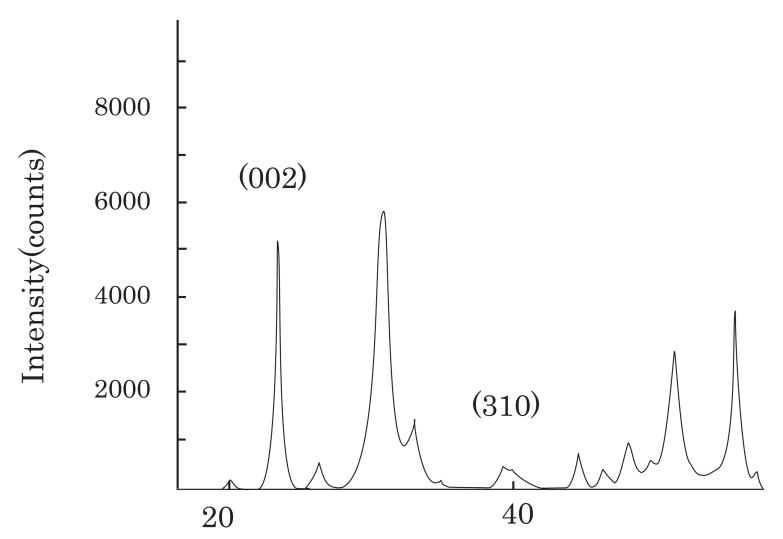

$2 \theta$ degree (b)

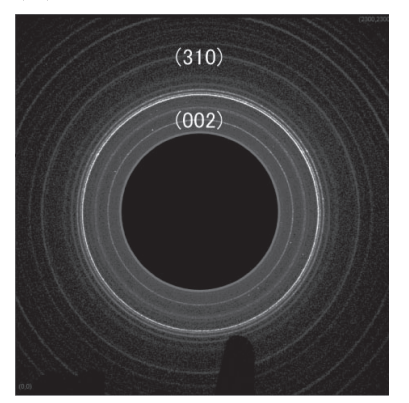

(c)

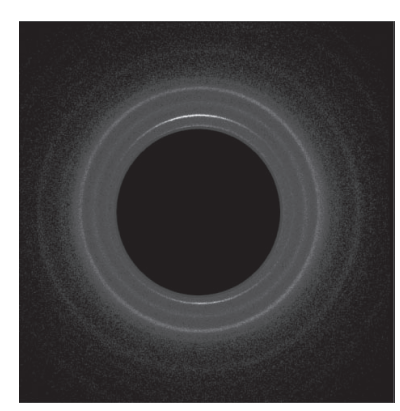

Fig. 6 Measurement of biological apatite crystal orientation: (a) X-ray diffraction spectrum sample of BAp: diffraction rings of (b) HAp and (c) BAp on the imaging plate (IP). X-ray diffraction data were evaluated by calculating the intensity ratio of the (002) and (310) diffraction peaks.

bone, respectively, located on the occlusal plane in the lower 1/6 (Fig. 4). Quantitative analysis of BAp crystallite alignment was done using a microbeam X-ray diffraction apparatus (RINT2500 + imaging plate; Rigaku, Tokyo, Japan) with 
both reflection and transmission optical systems employing a $\mathrm{Cu}-\mathrm{K} \alpha$ beam. The $\mathrm{X}$-axis in the mesiodistal direction, $\mathrm{Y}$-axis in the vertical direction to the occlusal plane, and $\mathrm{Z}$-axis in the buccolingual direction were set as the samples' axes (Fig. 5). For the X-ray diffraction systems, tube voltage was set at $40 \mathrm{kV}$ and tube current was set at $200 \mathrm{~mA}$. The incident beam was a small, circular beam of $100 \mu \mathrm{m}$ in diameter produced using a collimator. First, measurements were made in the direction of the $\mathrm{X}$-axis using the reflection optical system diffraction apparatus. During this time, diffracted X-ray beams were detected using curved position sensitive proportional counter. Measurements in the direction of the $\mathrm{Y}$ and $\mathrm{Z}$-axes were made using the transmission optical system diffraction apparatus. Measurement conditions were set according to the method developed by Nakano et $a l^{19)}$. The transmission diffraction apparatus rendered diffraction ring images on an imaging plate (IP) according to diffraction lines. The degree of BAp crystallite alignment was evaluated by reading the IP with detection software (3DS; Rigaku) and calculating the intensity ratio of the two diffraction peaks (002) and (310) (Fig. 6).

\subsection{Statistical analysis}

The means of each measurement at the five measurement points on the $\mathrm{X}, \mathrm{Y}$ and $\mathrm{Z}$-axes, respectively, were calculated and the Kruskal-Wallis test was performed to identify any dif- ferences. If a significant difference was detected, Steel's multiple comparison test was performed to identify between which groups a significant difference emerged. A $P$ value of less than 0.05 was considered statistically significant.

\section{Results}

Figure 7 shows the BAp crystallite alignment in the direction of the $\mathrm{X}$-axis at the five points measured using the reflection diffraction apparatus in each of the four groups (Dentate, Partially edentulous, $3 \mathrm{M}$ and $12 \mathrm{M}$ ). The diffraction intensity ratio of $(002) /(310)$ of the control substance hydroxyapatite powder was 1.51 . At point II, the $12 \mathrm{M}$ Group scored significantly higher than all other groups for BAp crystallite alignment in the direction of the X-axis. At point III, the Dentate Group and the $12 \mathrm{M}$ Group scored significantly higher than other groups for BAp crystallite alignment in the direction of the $\mathrm{X}$-axis.

Figures 8 and 9 show BAp crystallite alignment in the direction of the $\mathrm{Y}$ and $\mathrm{Z}$-axes measured at the five points using the transmission diffraction apparatus. The diffraction intensity ratio of (002)/(310) of the control substance hydroxyapatite powder was 2.35 . At point I, the $12 \mathrm{M}$ Group scored significantly higher for alignment in the direction of the Z-axis. At point II, the Dentate Group scored significantly higher for alignment in the direction of the Z-axis. At point III, the Den-

\section{BAp orientation along X-axis}

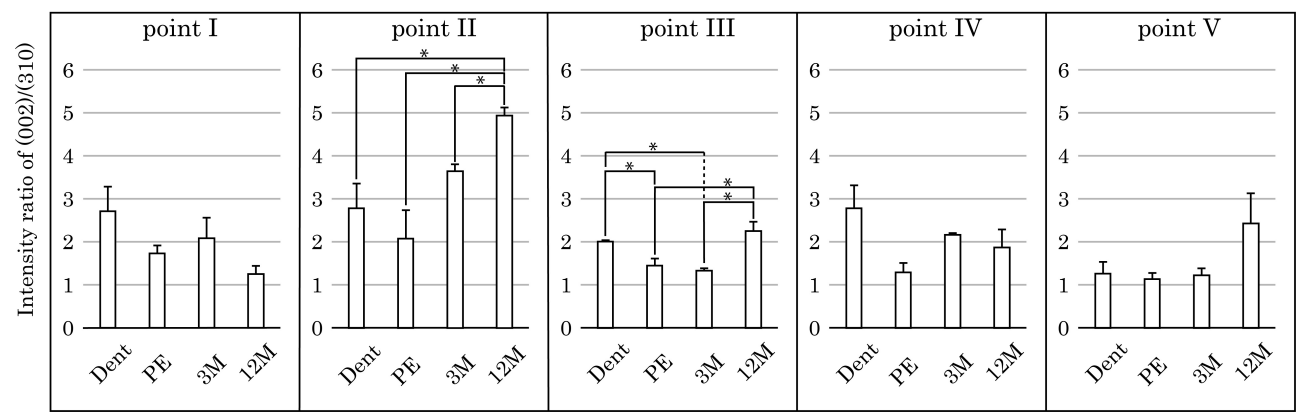

Fig. 7 BAp crystallite alignment in the direction of the $\mathrm{X}$-axis at each measurement point $(* P<0.05)$. Dent: Dentate PE: Partially edentulous. Values are means \pm the standard deviation.

\section{BAp orientation along Y-axis}

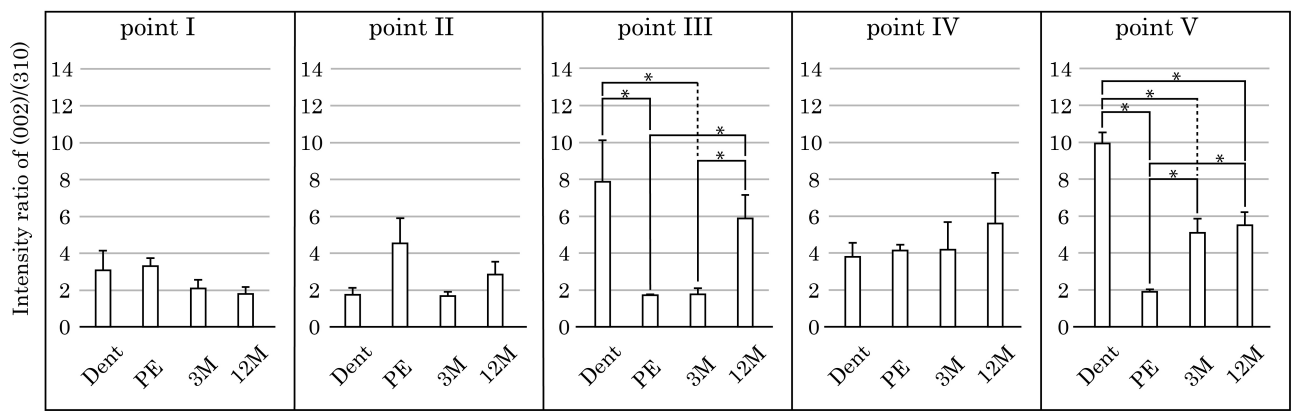

Fig. 8 BAp crystallite alignment in the direction of the Y-axis at each measurement point $(* P<0.05)$. Dent: Dentate PE: Partially edentulous. Values are means \pm the standard deviation. 


\section{BAp orientation along Z-axis}

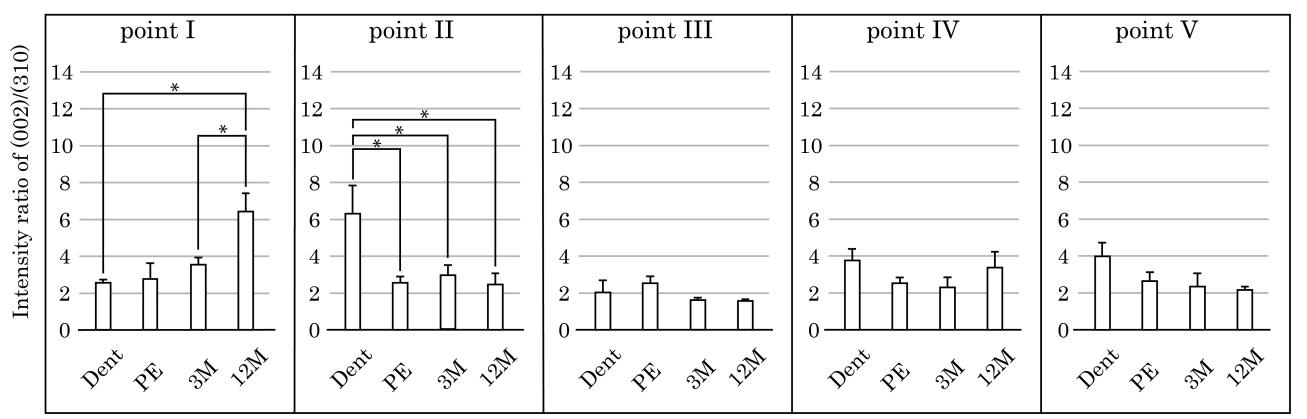

Fig. 9 BAp crystallite alignment in the direction of the Z-axis at each measurement point $(* P<0.05)$. Dent: Dentate PE: Partially edentulous. Values are means \pm the standard deviation.

tate Group and the 12M Group scored significantly higher for alignment in the direction of the Y-axis. At point IV, no significant differences were seen between any of the groups. At point $\mathrm{V}$, the Dentate Group scored significantly higher than all other groups for alignment in the direction of the Y-axis. In addition, the $3 \mathrm{M}$ and $12 \mathrm{M}$ Groups scored significantly higher than the Partially edentulous Group for alignment in the direction of the Y-axis.

\section{Discussion}

BAp crystallite alignment tends to improve with mechanical stress and growth, and unlike BMD, has been reported to conform sensitively to local stress distribution. Nakano et al. demonstrated through experiments in monkey jawbones that the BAp crystallite basically aligns in the mesiodistal direction of the long axis, but reported that the crystallite aligns in the direction of occlusal pressure as a result of masticatory pressure in the vicinity of teeth ${ }^{19)}$. Meanwhile, Nagisa et al. examined BAp crystallite alignment in the alveolar and basal regions of rat jawbones, while Morioka et al. and Furuya et al. examined this in human jawbones, and reported the same results $^{20-22)}$. Following tooth extraction, the external form of the jawbone changes in response to multiple local and functional factors ${ }^{25}$. In the present experiment, no preferential alignment was seen in any direction at any of the sites in partially edentulous jawbones. The present findings are in agreement with the study by Iwata et al. which reported that preferential alignment in the alveolar regions is lost due to tooth extraction $^{23)}$. Another report found that in the cranium, tooth extraction not only affected alveolar region morphology but also orbital and nasal cavity morphology as well as zygomatic bone morphology ${ }^{26)}$. Tooth extraction may therefore also affect alignment in the infraorbital canal and nasal cavity floors in edentulous jaws.

Moreover, preferential alignment was seen at point $\mathrm{V}$ in the $3 \mathrm{M}$ Group, and at points I, II, III and V in the 12M Group. Preferential alignment at these sites may reflect BAp crystallite formed following implant placement. In the occlusal direction, preferential alignment was seen in the lower region of the buccal cortical bone in the Dentate, $3 \mathrm{M}$ and $12 \mathrm{M}$ groups, and also in the higher region of buccal cortical bone in the Dentate and $12 \mathrm{M}$ groups. According to a study by Cat- taneo et al., compressive stress applied to the maxillary molar region is primarily concentrated in the zygomatic alveolar crest $^{27)}$. This suggests that most of the vertical load applied to the maxillary teeth and implants was borne by the buccal cortical bone in the present experiment. On the other hand, no preferential alignment was seen in palatal cortical bone in the present experiment. As Gross et al. pointed out, strain trajectories of strain applied to maxillary dentition divide into a "V-shaped" pattern that distributes strain to the buccal and palatal sides ${ }^{28)}$. No significant preferential alignment was seen on the palatal side in the present study, but the trends in this region could be clarified by evaluating different coordinate axes.

While preferential alignment was seen in the $12 \mathrm{M}$ Group in the infraorbital canal floor, nasal cavity floor and upper region of the buccal cortical bone, it was not seen in the $3 \mathrm{M}$ Group. Gang et al. compared the mechanical properties of peri-implant bone immediately after and 21 days, 4 months and 8 months after implant placement and reported that the local hardness and elastic modulus of bone had recovered as a result of remodeling ${ }^{29)}$. Changes in bone quality of peri-implant bone in samples taken after long-term placement of a superstructure may therefore have indirectly affected stress transfer. Moreover, different preferential alignment was seen in the Dentate and $12 \mathrm{M}$ groups in the infraorbital canal and nasal cavity floors. Sedat et al. reported that stress accumulation in bone tissue in a tooth-supported model was less than in an implant-supported model ${ }^{30)}$. It can be presumed that the difference in mechanical property between periodontal and peri-implant tissues may explain the stress accumulation reported in the present study. Also, considering the report by Ohashi et al., the alignment seen in the 12M Group suggests that implant placement altered the mechanical environment $^{24)}$. Frost et al. proposed the mechanostat theory for bone modifications that occur as a result of applying appropriate stress $^{31)}$. In effect, the samples taken from the Dentate, $3 \mathrm{M}$ and $12 \mathrm{M}$ groups may have changed as a result of bone nanostructure optimizing itself in response to the masticatory load. In addition, Kan reported that bone resorption due to pathological overload can be avoided through dissipation to distant sites of strain caused by functional pressure applied to implants ${ }^{32)}$. The mechanical environmental changes suggested by the present experiment indicate that the decline of bone 
quality in peri-implant tissue could be prevented. Therefore, BAp crystallites in peri-implant bone should be aligned in the direction of local compressive stress for long-term survival of implants.

\section{Conclusion}

The structural changes caused by dental implant treatment in peri-implant jawbone not only occur at sites in contact with the implant, but also in distant sites. These changes indicate that the alignment of BAp crystallites responded to the functional pressure applied to the implant. Therefore, clinicians should choose implant placement sites in consideration of the effects of occlusal loading on the surrounding jawbone.

\section{Acknowledgements}

This research was supported by a Grant-in-Aid for Scientific Research (Challenging Exploratory Research: 25463055) from the Japan Society for the Promotion of Science. The authors would like to thank Mrs. Eiko Watanabe for her technical assistance.

\section{REFERENCES}

1) H. Wada, J. Hosomichi, Y. Shimomoto and K. Soma: Orthod wave. 67 (2008) 9-14.

2) F. Bassi, M. Procchoio, C. Fava, G. Schierano and G. Preti: Clin. Oral Implants Res. 10 (1999) 356-361.

3) V.J. Kingsmill and A. Boyde: J. Anat. 192 (1998) 233-244.

4) S. Matsunaga, Y. Shirakura, T. Ohashi, K. Nakahara, Y. Tamatsu, N. Takano and Y. Ide: Int. J. Prosthodont. 23 (2010) 333-338.

5) T. Albrektsson, P.I. Brånemark, H.A. Hansson and J. Lindström: Acta Orthop. Scand. 52 (1981) 155-170.

6) T. Traini, B. Assenza, F. San Roman, U. Thams, S. Caputi and A. Piattelli: Clin. Oral Investig. 10 (2006) 151-156.

7) P. Leucht, J.B. Kim, R. Wazen, J.A. Currey, A. Nanci, J.B. Brunski and J.A. Helms: Bone 40 (2007) 919-930.

8) K. Vandamme, I. Naert, L. Geris, J.V. Sloten, R. Puers and J. Duyck:
Clin. Oral Implants Res. 18 (2007) 471-480.

9) J. Duyck, K. Vandamme, L. Geris, H. Van Oosterwyck, M. De Cooman, J. Vandersloten, R. Puers and I. Naert: Arch. Oral Biol. 51 (2006) 1-9.

10) L. Geris, K. Vandamme, I. Naert, J. Vander Sloten, J. Duyck and H. Van Oosterwyck: J. Biomech. 41 (2008) 145-154.

11) J. Irish, A.S. Virdi, K. Sena, M.A. McNulty and D.R. Sumner: J. Orthop. Res. 31 (2013) 800-806.

12) J. Duyck, H.J. Rønold, H. Van Oosterwyck, I. Naert, J. Vander Sloten and J.E. Ellingsen: Clin. Oral Implants Res. 12 (2001) 207-218.

13) P. Asikainen, E. Klemetti, T. Vuillemin, F. Sutter, V. Rainio and R. Kotilainen: Clin. Oral Implants Res. 8 (1997) 465-468.

14) F. Isidor: Clin. Oral Implants Res. 7 (1996) 143-152.

15) S. Kirschner: Wurzbg. Medizinhist. Mitt. 22 (2003) 67-80.

16) D.B. Burr: J. Musculoskelet. Neuronal Interact. 2 (2002) 201-204.

17) NIH Consensus Development Panel on Osteoporosis Prevention, Diagnosis, and Therapy: JAMA 285 (2001) 785-795.

18) J.C. Elliot: Structure and Chemistry of the Apatites and Other Calcium Orthophosphates, (Elsevier Science, Amsterdam, 1994) pp. 1-389.

19) T. Nakano, K. Kaibara, Y. Tabata, N. Nagata, S. Enomoto, E. Marukawa and Y. Umakoshi: Bone 31 (2002) 479-487.

20) N. Nagisa, T. Nakano, N. Hashiguchi, W. Fujitani, Y. Umakoshi and M. Shimahara: Oral Sci Int. 7 (2010) 19-25.

21) T. Morioka, S. Matsunaga, M. Yoshinari, Y. Ide, T. Nakano, H. Sekine and Y. Yajima: Cranio 30 (2012) 32-40.

22) H. Furuya, S. Matsunaga, Y. Tamatsu, T. Nakano, M. Yoshinari, Y. Ide and S. Abe: Mater. Trans. 53 (2012) 980-984.

23) M. Iwata, S. Matsunaga, T. Morioka, T. Nakano, S. Abe, M. Yoshinari and Y. Yajima: J Hard Tissue Biol. 24 (2015) 235-240.

24) T. Ohashi, S. Matsunaga, K. Nakahara, S. Abe, Y. Ide, Y. Tamatsu and N. Takano: Clin. Oral Investig. 14 (2010) 507-513.

25) V.J. Kingsmill: Crit. Rev. Oral Biol. Med. 10 (1999) 384-404.

26) S. Candice, B. Desiré and H. Jason: Int. J. Legal Med. 130 (2016) 223243.

27) P.M. Cattaneo, M. Dalstra and B. Melsen: Am. J Orthod Dentofac. 123 (2003) 367-373.

28) M.D. Gross, G. Arbel and I. Hershkovitz: J. Oral Rehabil. 28 (2001) 684-694.

29) W. Gang, Q. Xinhua and Y. Zhifeng: PLoS ONE 9 (2014) e108324.

30) S. Guven, K. Beydemir, S. Dundar and V. Eratilla: Eur J. Dent. 9 (2015) 329-339.

31) H.M. Frost: Anat. Rec. 219 (1987) 1-9.

32) J.P. Kan, R.B. Judge and J.E. Palamara: Clin. Oral Implants Res. 25 (2014) e73-e82. 